\title{
Meninas na Computação: uma análise inicial da participação das mulheres nos cursos de Sistemas de Informação do estado de Alagoas
}

\author{
José Vitor de Abreu Silva ${ }^{1}$, Letícia Rocha de Oliveira1, André Almeida Silva ${ }^{1}$ \\ Unidade de Ensino de Penedo - Universidade Federal de Alagoas (UFAL) \\ Av. Beira Rio S/N, Centro - 57200-000 - Penedo - AL- Brasil \\ vitorabreeus@gmail.com, leticia.roch@hotmail.com, \\ andre.almeidaearapiraca.ufal.br
}

\begin{abstract}
The significant difference between the percentage of men and women in Computer-related courses Is remarkable. In Order to understand the reasons for the low number and high degree of evasion of girls enrolled in this area, studies and projects have been developed, outlining actions to add more women to the field of technology. Thus, this article presents the levels of female participation in the Information Systems courses of the state of Alagoas, considering the number of entrantmen and the enrolled students, which results in very low percentages when compared to the number Of men in this field. From the initial results, intervention initiatives are proposed, aimed at attracting, retaining and increasing the representativeness of the female gender in Computing, discussing the professional and social equity between genders, in addition to delineating the Next steps of this work.

Resumo. É notável a significativa diferença entre o percentual de homens e mulheres nos cursos relacionados à Computação. Visando compreender os motivos do baixo número e do alto grau de evasão de meninas matriculadas nesta área, estudos e projetos vêm sendo desenvolvidos, traçando ações para somar mais mulheres ao campo da tecnologia. Assim, este artigo apresenta os níveis de participação feminina nos cursos de Sistemas de Informação do estado de Alagoas, considerando o número de ingressantes e o de estudantes matriculadas, o qual resulta em porcentagens muito baixas quando comparadas ao número de homens neste campo. A partir dos resultados iniciais, são propostas iniciativas de intervenção, voltadas a atrair, reter e aumentar a representatividade do gênero feminino na Computação, discutindo-se acerca da equidade profissional e social entre os gêneros, além de delinear as próximas etapas deste trabalho.
\end{abstract}

\section{Introdução}

Permeia na sociedade a necessidade da equiparação de gênero e do avanço contra o machismo, que ainda é bastante marcante nos diversos setores e mercado. Muitas vezes, a não participação das mulheres em alguns serviços e áreas, é justificada pelo padrão préestabelecido de que os homens se interessam mais por determinado contexto e, dessa forma, a baixa presença de mulheres em alguns campos, sobretudo àqueles ligados à Tecnologia da Informação e às Ciências Exatas, como um todo, é vista como natural. 
Para Epstein (2007), a maior divisão social que caracteriza o mundo atual é a divisão sexual. Mesmo diante das indiscutíveis conquistas das mulheres na educação e no trabalho, assim como sua participação efetiva e crescente em diversas áreas de conhecimentos, que até meados do século XX seria inimaginável discutir a possibilidade de inserir-se, o gênero feminino ainda é preterido no mercado de trabalho e, muitas vezes, desvalorizado quando se comparam os ganhos de homens e mulheres que exercem as mesmas funções.

A desigualdade entre gêneros é considerada um desperdício que afeta toda a sociedade. Neste contexto, destaca-se que "deixar as mulheres para trás", significa não somente desprezar as importantes contribuições que as mulheres trazem para a economia, mas também desperdiçar anos de investimento em educação de meninas e jovens mulheres" (OECD, 2012, p.2).

Apesar da disparidade entre homens e mulheres nos setores da sociedade ser gritante, nota-se que as meninas vêm ganhando cada vez mais espaço no setor de tecnologia. Na maioria das vezes, a área de exatas, que perpassa das engenharias até o meio tecnológico, é pouco popular a representação feminina, embora seja historicamente visto que as mulheres são as pioneiras nesse campo. Todavia, atualmente estão sendo criadas várias iniciativas que apoiam e incentivam o trabalho de mulheres com tecnologia, como salienta uma pesquisa realizada pelo LinkedIn (2017) que mostra aumento de $18 \%$ na quantidade de mulheres que dominam cargos de liderança no setor de tecnologia entre os anos 2008 e 2016.

Diante dos diversos artigos encontrados acerca dos temas mulheres na computação e mulheres nas exatas, oriundos de pesquisas bibliográficas, considera-se importante realizar neste trabalho um mapeamento referente ao número de ingressantes nos cursos de graduação em Sistemas de Informação (SI) de Alagoas. O estado conta com três cursos de bacharelado na área de Computação em Instituições de Ensino Superior (IES) da rede pública, os quais são: Ciência da Computação, Engenharia da Computação e Sistemas de Informação. Por conta do vínculo dos autores deste artigo em SI, bem como pelo interesse em estudar o curso, foi enfatizado neste trabalho o bacharelado em Sistemas de Informação, com o objetivo de traçar um perfil básico deste público, com a finalidade de idealizar iniciativas de intervenção, voltadas a atrair, reter e aumentar a representatividade do gênero feminino nesta área.

O método utilizado para a elaboração do artigo foi o exploratório-descritivo, iniciado com revisão bibliográfica, e dando continuidade com a pesquisa de natureza documental, sendo especificado melhor na Seção 3. Por conseguinte, têm-se destaque e a comprovação através de dados empíricos de que o percentual de garotas que ingressam nos cursos de SI do estado é bem menor que o número de meninos matriculados, com detalhamento nas Seções 4 e 5 deste artigo.

\section{História das Mulheres na Computação}

\subsection{De maioria à raridade nos cursos de informática}

Nas últimas décadas, as discussões referentes à desigualdade de direitos, considerando questões de gênero, acentuaram-se. Mesmo persistindo enormes desigualdades, houve importantes ganhos no direcionamento da equidade raça e gênero. Mas, ainda hoje as mulheres são discriminadas e têm suas competências colocadas em xeque, assim como 
aponta Rapkiewicz (1998) ao relatar que elas são consideradas inábeis para as áreas científicas, preconceito que se estende para campo das tecnologias.

Porém, apesar de toda a discriminação referenciada, nota-se que as mulheres foram elementares para o atual estágio de desenvolvimento da informática. Basta analisar os estudos, trabalhos e inventos realizados pelos pioneiros da área, que se destacará a fundamental participação feminina na evolução da informática, fato que muitas vezes parece se tornar esquecido, invisibilizando o papel e importância das cientistas.

Augusta Ada King (1815 - 1852), a Lady Lovelace, é um grande exemplo das precursoras da informática. De acordo com Toole (1998), matemática, fillha do poeta inglês Lord Byron e da também matemática Ann Isabella Milbank, Ada é considerada a primeira mulher a programar na história. Outro nome de destaque é o de Grace Murray Hopper (1906-1992) que, segundo Maisel (2000), formou-se em matemática e física e contribuiu para o desenvolvimento da linguagem de programação COBOL na década de 1950 e ainda está em uso, sendo também responsável pelo desenvolvimento do primeiro compilador. Inclusive, o termo usual da computação "bug" foi totalmente creditado a Grace, ao encontrar a causa do erro no computador em que estava programando: literalmente um inseto.

É importante ainda fazer menção ao ENIAC, desenvolvido por John Mauchly e J. Presper Eckert, o primeiro computador eletrônico do mundo. Esta máquina, de acordo com Gürer (2002), foi inicialmente programada por seis mulheres que compunham o Corpo Voluntário Feminino para Emergências (WACS) durante a Segunda Guerra Mundial: Kathleen McNulty Mauchly Antonelli, Jean Jennings Bartik, Fraces Synder Holberton, Marlyn Wescoff Melzer, Fraces Bilas Spence e Ruth Lichterman Teitelbaum.

A carência de modelos femininos, ou melhor, a não divulgação dos nomes que fizeram e fazem diferença nas ciências, tem perpetuado o pensamento de que mulheres não possuem aptidão para o meio científico e tecnológico, fazendo com que fique cada vez mais escassa a participação feminina nas ciências, principalmente nas exatas. À vista disso, para a computação, inclusa nas ciências exatas, é também relevante resgatar a história da participação vital que as cientistas tiveram para com a evolução da informática.

A história das pioneiras evidencia, de fato, o papel basilar no desenvolvimento da informática, porém, os seus nomes são pouco lembrados ou nem mesmo mencionados em grande parte dos cursos e eventos da área, situação que vem mudando timidamente com o tempo neste cenário de acentuada disparidade entre os gêneros na Computação, trazendo reflexos na produção científica e acadêmica. De tal modo, considerando o contexto do pioneirismo na informática, as mulheres têm um elevado valor na informática, pois a atuação fora imprescindível.

Assim, ressaltar o contexto histórico do poder feminino na informática deve/deveria contribuir para o aumento de meninas na área, sendo essa uma das principais bandeiras levantadas. Tabak (2002, p.57) expõe que "a imagem da Ciência como ocupação masculina, a crença de que a mulher teria uma competência inferior à do homem, agiriam como barreiras na construção de uma carreira bem-sucedida no mundo científico". Indo além das palavras da supracitada autora, infere-se que além de impedir o progresso das carreiras, toda a ciência sai prejudicada a partir do momento que mentes femininas talentosas para a Computação são desperdiçadas e, em alguns casos, sequer descobertas. 


\subsection{Uma visão feminina da mulher na computação}

Tem sido uma constante nas últimas décadas a busca de igualdade de direitos e oportunidades, visando à eliminação das disparidades existentes entre homens e mulheres. Conforme mencionado, a atual realidade já apresenta muitas mudanças positivas nesta direção. Contudo, em determinados setores, como na área de tecnologia de informação, ainda se tem muito a progredir no tocante à equidade.

Em um levantamento realizado no ano de 2019, a empresa de consultoria Yoctoo (2019), especializada em realizar recrutamento de profissionais de TI, apontou que $82,8 \%$ das mulheres entrevistadas relata ter vivido, ou ainda vivenciar, preconceito de gênero dentro do seu ambiente de trabalho. Em relação à área acadêmica, 61,8\% assegura ter vivido ou vivencia este preconceito.

O estudo realizado pela empresa de consultoria é bastante revelador, trazendo outros importantes números: $91 \%$ das entrevistadas afirmam que ainda existe preconceito dentro das empresas e que essas ainda estão dando os primeiros passos direcionados à implementação de políticas de diversidade e inclusão; $72 \%$ afirmam que o ambiente familiar não costuma estimular meninas a gostarem de brincadeiras ou carreiras ligadas à tecnologia; $42 \%$ das participantes afirmam que o maior desafio é ter de provar a todo tempo que são profissionais competentes; e 36\% diz ser urgente ações que incentivem o interesse pela tecnologia começando pela infância.

Dessa forma, analisando a pesquisa da Yoctoo (2019), nota-se que duas questões são citadas como possíveis justificativas à baixa representatividade e interesse feminino no nicho da tecnologia, que são: a falta de incentivo na infância e a ausência de reconhecimento tanto no âmbito profissional quanto no acadêmico. Esses pontos advêm de fatos históricos vistos com maior ênfase no último século, quando, como ressalta Schwartz et. al (2006), "as atividades para a manutenção da família passaram a fazer parte da esfera privada, sendo atribuído este espaço às mulheres" e a ciência foi direcionada à esfera pública, vista como campo atribuído aos homens, fato que excluiu a mulher das universidades e do meio científico.

Em uma sociedade cada vez mais tecnológica, analisar e perceber esse déficit é preocupante, pois quanto mais diversidade houver entre as equipes, seja na ciência ou no mercado, mais completas e precisas serão as soluções criadas. Schiebinger (2001, p.37) destaca que a ciência moderna é fruto de anos de exclusão feminina e que "o processo de trazer mulheres para a ciência exigiu, e vai continuar a exigir, profundas mudanças estruturais na cultura, métodos e conteúdo da ciência”.

Alguns estudos voltam-se a analisar as meninas na Computação e aqui merece destaque, o trabalho de Moreira, Silva \& Carvalho (2018), que mostra uma prospecção da presença feminina em cursos de Ciência da Computação e constatam que o número de mulheres na área reduz-se a uma taxa de $0,4 \%$ ao ano e que, sem intervenções para reverter essa tendência, não haverá mais mulheres em 2050.

Logo, são necessárias ações bem pensadas desde a educação básica, a fim de diminuir a distância entre homens e mulheres na ciência e, consequentemente, no setor profissional. No recorte dado neste trabalho, as iniciativas voltadas à inclusão de meninas na área da Computação, estudando o curso de Sistemas de Informação, terão maior ênfase, pois corresponde ao objeto de estudo. 


\section{Materiais e Métodos}

Este trabalho foi realizado através de estudo preliminar do projeto de extensão Garotas na TI: despertando vocações e descobrindo talentos, que está em processo de registo na Universidade Federal de Alagoas (UFAL) - Campus Arapiraca - Unidade de Ensino de Penedo. O projeto conta com a participação de estudantes e professores do curso de Sistemas de Informação dessa unidade.

O método para a elaboração do presente artigo é o exploratório-descritivo, iniciado com revisão bibliográfica, conforme assinalado na seção anterior, que possibilitou a construção de referencial teórico sobre assuntos relacionados à mulher na computação. Em seguida, foi feita pesquisa de natureza documental, coletando-se dados nas páginas oficiais dos cursos de Sistemas de Informação das instituições públicas de ensino que oferecem SI no estado de Alagoas: Universidade Federal de Alagoas (UFAL), em seus Campus Aristóteles Calazans Simões (A.C. Simões) e de Arapiraca; e Instituto Federal de Educação, Ciência e Tecnologia de Alagoas (IFAL), em seus Campus Maceió e Arapiraca. Buscou-se confirmar a hipótese de que há disparidade de gênero na área da Computação, especificamente em Sistemas de Informação, curso que é foco deste trabalho.

Logo após o recolhimento dos dados, procedeu-se análise sob os mesmos, organizando-os por meio de planilhas eletrônicas e gerando gráficos para uma melhor visualização dos números que foram encontrados. Então, com o estudo realizado, são propostas iniciativas de intervenção, voltadas à atração, retenção e aumento da representatividade feminina na Computação, pensando-se no curso de SI, discutindo-se ainda sobre equidade profissional e social entre os gêneros, delineando assim as próximas etapas do projeto de extensão que originou este trabalho.

\section{Resultados e Discussão}

Esta seção se concentra na disposição e breve interpretação dos dados recolhidos através de pesquisas realizadas nos sites da Universidade Federal de Alagoas e do Instituto Federal de Alagoas, sendo estas instituições as únicas que disponibilizam os cursos de Sistemas de Informação na rede pública de ensino superior de Alagoas. Ademais, ainda nesta seção, serão sugeridas as propostas de intervenção anteriormente referidas. Ressalta-se que os dados que originaram a Figura 1 e a Figura 2 advêm da página oficial da UFAL ${ }^{1}$ e os dados da Figura 3 e da Figura 4 são originados da página do IFAL ${ }^{2}$.

\subsection{Dados do Bacharelado em SI da UFAL - Arapiraca - Penedo}

O primeiro curso analisado foi da Unidade de Ensino Penedo, Campus Arapiraca, da UFAL. Foi constatado que no último ano de ingresso, 2018, das 50 vagas disponibilizadas, apenas 12 mulheres preencheram em todas as demandas existentes (ampla concorrência e sistemas de cotas), o que equivale a $24 \%$ do total de vagas oferecidos, contra 38 homens matriculados. A Figura 1 ilustra esses dados.

\footnotetext{
${ }^{1}$ UFAL. Disponível em: https://ufal.br/. Acesso em: fev. 2019.

2 IFAL. Disponível em: https://www2.ifal.edu.br/. Acesso em: fev. 2019.
} 


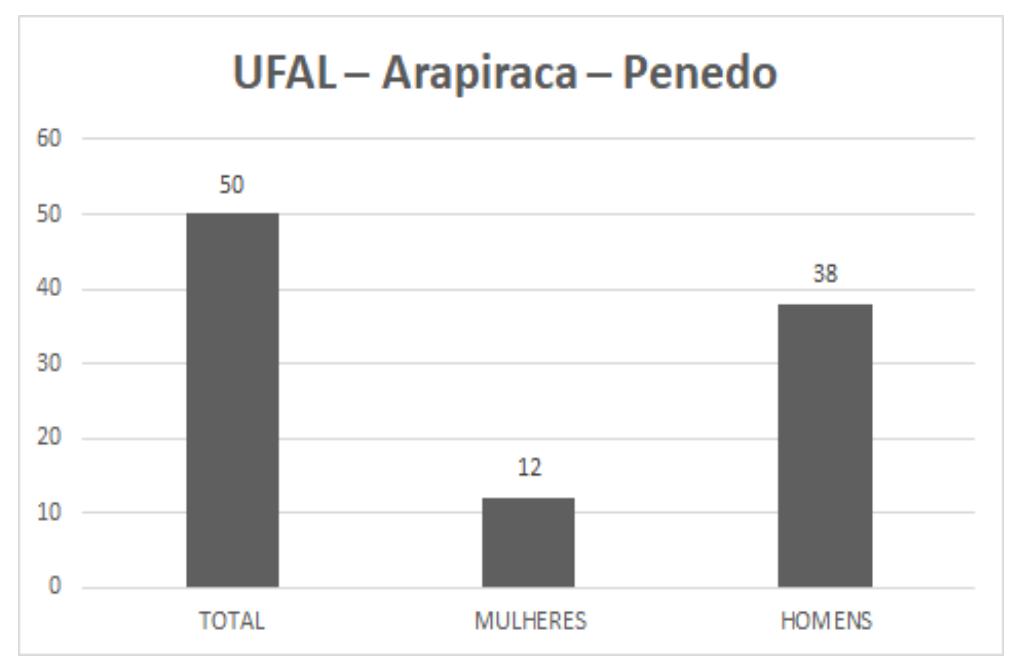

Figura 1. Quantidade de homens e mulheres no curso de Sistemas de Informação Unidade de Ensino Penedo, 2018.

\subsection{Dados do Bacharelado em SI da UFAL - Campus A.C. Simões}

Também foi analisado o único curso na modalidade de Educação a Distância (EAD) de Sistemas de Informação de Alagoas que é ofertado pelo Campus A.C. Simões da UFAL. O quantitativo visto seguiu a linha do curso da Unidade de Ensino Penedo. Neste caso, a instituição oferece 60 vagas semestrais que foram preenchidas por 12 mulheres no período 2018.2 apenas, valor equivalente a $20 \%$ das vagas totais, contra 48 homens matriculados, como mostra a Figura 2.

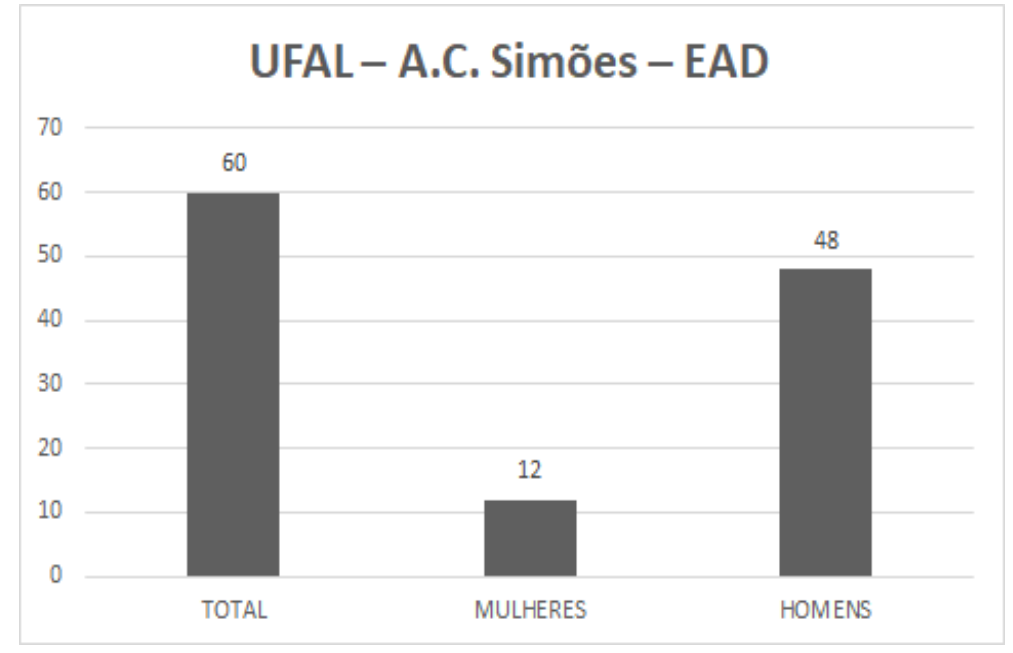

Figura 2. Quantidade de homens e mulheres no curso de Sistemas de Informação do Curso EAD da UFAL, 2018.2.

\subsection{Análise IFAL - Arapiraca}

Logo após a análise dos cursos da UFAL, partiu-se para o IFAL, onde a presença feminina no Campus de Arapiraca não foi diferente. O curso é novo e teve sua primeira oferta de vagas no ano de 2019. Foram oferecidas 40 vagas, das quais somente 5 foram preenchidas por mulheres e 35 por homens. O número de meninas matriculadas equivalente a $12,5 \%$ das vagas totais. A Figura 3 ilustra esses dados. 


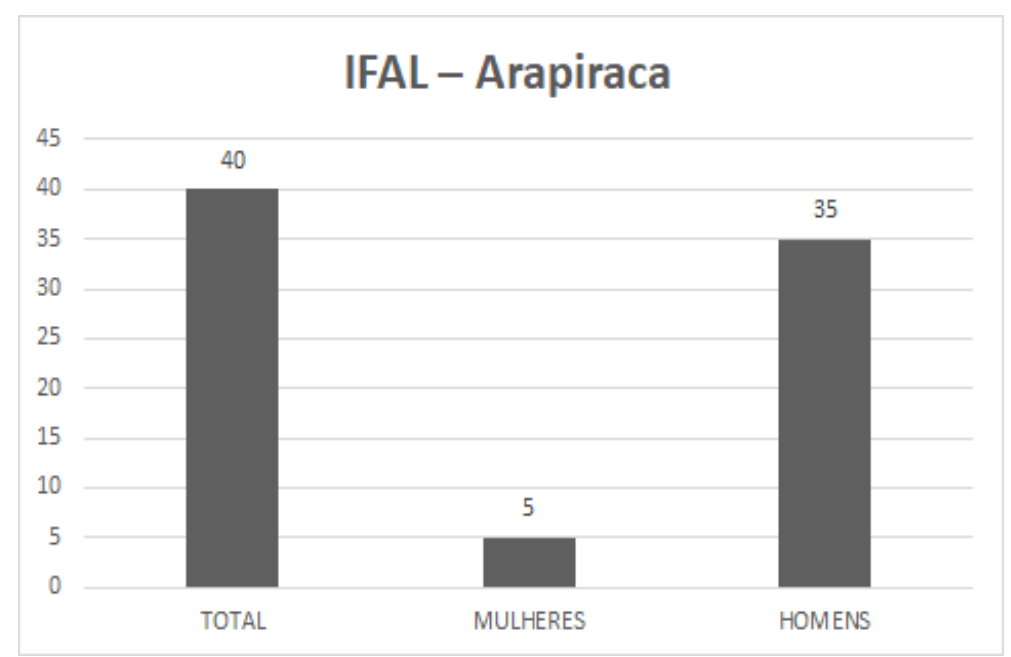

Figura 3. Quantidade de homens e mulheres no curso de Sistemas de Informação do Instituto Federal de Alagoas, Campus Arapiraca, 2019.1.

\subsection{Análise IFAL - Maceió}

Por último, foi analisado o curso ofertado pelo Campus de Maceió do IFAL que, no total de 23 vagas, 8 foram preenchidas por mulheres e 15 por homens, o que equivale a $34,8 \%$ de mulheres entre as vagas totais, conforme mostrado na Figura 4.

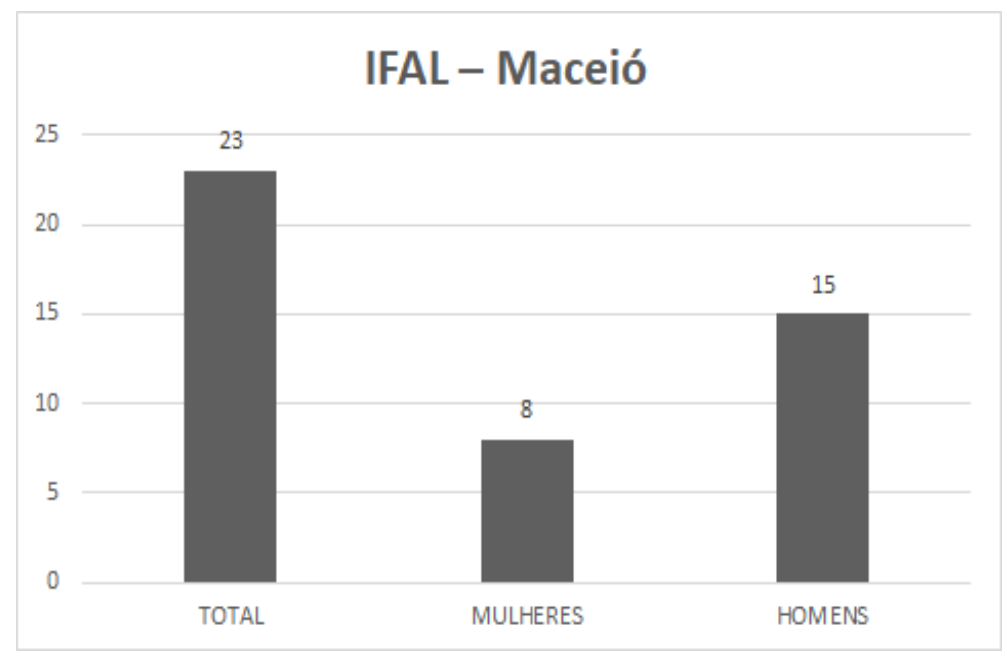

Figura 4. Quantidade de homens e mulheres no curso de Sistemas de Informação do Instituto Federal de Alagoas, Campus Maceió, 2018.2.

\subsection{Propostas de intervenção}

Com base nos resultados das pesquisas, pôde-se perceber que os dados dispostos possuem o mesmo viés das colocações dispostas na Seção 2, quando foi ressaltada a baixa representatividade feminina na Ciência e, pelo destaque dado neste trabalho, na Computação. Dos 4 cursos analisados, em nenhum deles o número de mulheres foi equivalente ou superior ao número de homens.

Do total das 113 vagas ofertadas nos 4 Campus, apenas 37 delas foram preenchidas por mulheres, o que equivale a $32,74 \%$ do total de vagas. Considerando esse cenário, é importante organizar ou apoiar ações que objetivem aumentar a participação 
feminina na área de tecnologia, pois, apesar dos avanços, ainda se tem um problema real: o número de mulheres na tecnologia ainda é muito pequeno. Aqui não foi estudada a evasão de meninas na Computação, o que por si só configura outro problema e agrava ainda mais o baixo número delas na área.

Deste modo, levando em conta a necessidade de ser debatida e estimulada a inserção da mulher na Computação e sabendo que o curso de Sistemas de Informação, que relaciona duas importantes vertentes do mercado, a Gestão da Informação e a Programação, a ideia que originou este trabalho objetiva promover ações que ampliem a participação feminina na área da Computação, especialmente nos cursos de Sistemas de informação, dadas as características de estudantes e professores envolvidos no projeto que efetivaram as ações propostas. Tais ações têm como público alvo alunas de cursos pré-vestibulares e graduandas dos cursos de Sistemas de Informação do estado.

As atividades voltadas às alunas de cursos pré-vestibulares têm como objetivo apresentar o curso como uma possibilidade para que elas desenvolvam suas carreiras profissionais. Para as graduandas dos cursos, as ações têm como objetivo diminuir a evasão, retendo as estudantes e ampliando, assim, a participação feminina na academia e, consequentemente, também, no mercado. Salienta-se que a difusão do conhecimento será a principal estratégia para despertar vocações, descobrir novos talentos e apoiar as garotas que já fazem parte do quadro de estudantes de Sistemas de Informação.

\section{Considerações Finais}

Neste artigo foi apresentada uma análise inicial da participação das mulheres nos cursos de Sistemas de Informação do estado de Alagoas, considerando as instituições públicas de ensino superior do estado, que ofertam o referido curso. Através de pesquisa exploratório-descritivo, iniciado com revisão bibliográfica e seguido da análise de dados advindos da UFAL e do IFAL.

As primeiras conclusões são as de que, de fato, o percentual de garotas que ingressam nos cursos de SI do estado é bem menor que o número de meninos matriculados, seguindo a hipótese que fora criada com o estudo realizado na Seção 2 deste trabalho. Outra situação percebida, mas ainda não estudada de maneira sistematizada, corresponde a alta evasão que estes cursos enfrentam, sobretudo, do gênero feminino, fazendo com que o número de meninas que já é pouco, diminua cada vez mais. Ainda, buscando entender a baixa representatividade do gênero feminino em áreas de Ciências exatas com ênfase no curso de SI, torna-se importante para o meio acadêmico localizar e entender, também, IES privadas, com intuito de averiguar se há mudanças na equidade.

Diante dessa situação, analisando tanto os dados da pesquisa Yoctoo (2019) quanto os gerados pelos pesquisadores deste trabalho, bem como pensando no projeto de extensão, em atual estágio introdutório, Garotas na TI: despertando vocações $e$ descobrindo talentos, pretende-se realizar ações de propagação do conhecimento com dois públicos e direcionamentos: garotas dos cursos pré-vestibular, focando-se no caráter vocacional; e garotas que já estão cursando Sistemas de Informação, focalizando na permanência dessas meninas no curso.

Dessa forma, perante tudo o que foi mostrado, percebe-se que a mulher já teve muito mais representatividade nas áreas científicas, sendo, inclusive, precursoras de algumas delas. Hoje, porém, enfrentam grandes obstáculos na sociedade cada vez mais desigual e preconceituosa. Assim, traçar formas de aumentar o número de meninas de 
Sistemas de Informação e na Computação, como um todo, é proporcionar maior diversidade à área, contribuindo para melhores discussões, soluções e com uma sociedade mais equânime e justa.

\section{Referências}

Epstein, C. (2007) Great divides: the cultural, cognitive, and social bases of the global subordination of women. American Sociological Review, v.12, Fev, p.1- 25.

Gomes, W. (2014) Incentivando meninas do ensino médio à área de Ciência da Computação usando Scratch como ferramenta. n1, p. 1-10.

Gürer, Denise. (2002) Women in Computing History. In: ACM SIGCSE Bulletin, vol. 34, $\mathrm{n}^{\mathrm{o}}$ 2, California, 2002, pp.116-120.

Linkedin. (2017) International Women's Day: Celebrating the Progress of Women Leaders in the Workplace. Disponível em: https://blog.linkedin.com/2017/march/6/international-womens-day-trends-in-womenhiring-and-leadership-linkedin. Acesso em: fev. 2019.

Maisel, Merry. (2000) Tribute to Grace Murray Hopper. Grace Hopper Celebration of Women in Computing. Disponível em: http://www. sdsc.edu/Hopper/GHC_INFO/hopper.html. Acesso em: fev. 2019.

Moreira, J., Silva, R., \& Carvalho, M. (2018). Cenários Prospectivos: Uma Visão do Futuro da Presença Feminina em Cursos de Ciência da Computação de uma Instituição de Ensino Superior. In $26^{\circ}$ Workshop sobre Educação em Computação (WEI 2018). Porto Alegre: SBC.

OECD. (2012) Education at glance 2012: OECD Indicators. Disponível em: http://dx.doi. org/10.1787/eag-2012-em. Acesso em: fev. 2019.

Oliveira, A. (2014) Perfil Feminino em Computação: Análise Inicial. XXXIV Congresso da Sociedade Brasileira de Computação. n1, p. 1465-1474.

Rapkiewicz, Clevi Elena. Femina Computationalis ou A construção do Gênero na Informática. Tese de Doutorado em Ciências em Engenharia de Sistemas e Computação, UFRJ, Rio de Janeiro, 1998.

Schiebinger, Londa. (2001) O feminismo mudou a ciência? Bauru-SP, EDUSC.

Schwartz, Juliana; Casagrande, Lindamir Salete; Leszczynski, Sonia Ana Charchut and Carvalho, Marilia Gomes de. (2006) Mulheres na informática: quais foram as pioneiras?. Cad. Pagu [online]. 2006, n.27, pp.255-278.

Tabak, Fanny. (2002) O laboratório de Pandora: Estudos sobre a ciência no feminino. Rio de Janeiro, Garamond.

Toole, Betty Alexandra. (1998) Ada Lovelace Biography. Disponível em: http://www.well.com/user/adatoole/bio.htm. Acesso em: fev. 2019.

Yoctoo. (2019) Yoctoo realiza pesquisa exclusiva para entender quais os desafios nas carreiras das mulheres em tecnologia. Disponível em: https://www.yoctoo.com/en/blog/2019/03/yoctoo-realiza-pesquisa-exclusiva-paraentender-quais-os-desafios-nas-carreiras-das-mulheres-em-tecnologia. Acesso em: fev. 2019. 